\title{
Link Invariants for Flows in Higher Dimensions
}

\author{
Hugo García-Compeán! \\ Departamento de Física, Centro de Investigación y de Estudios Avanzados del IPN \\ P.O. Box 14-740, 07000 México D.F., México
}

\begin{abstract}
Linking numbers in higher dimensions and their generalization including gauge fields are studied in the context of $B F$ theories. The linking numbers associated to $n$-manifolds with smooth flows generated by divergence-free $p$ vector fields, endowed with an invariant flow measure are computed in different cases. They constitute invariants of smooth dynamical systems (for non-singular flows) and generalizes previous results for the 3-dimensional case. In particular, they generalizes to higher dimensions the Arnold's asymptotic Hopf invariant for the three-dimensional case. This invariant is generalized by a twisting with a non-abelian gauge connection. The computation of the asymptotic Jones-Witten invariants for flows is naturally extended to dimension $n=2 p+1$. Finally we give a possible interpretation and implementation of these issues in the context of string theory.
\end{abstract}

August, 2009

\footnotetext{
${ }^{1}$ e-mail: compean@fis.cinvestav.mx

${ }^{2}$ e-mail: rsantos@fis.cinvestav.mx
} 


\section{Introduction}

Since their gestation topological field theories have been used to describe topological invariants of manifolds in various dimensions. Cohomological field theories are quantum field theories whose states are obtained from a BRST-like symmetry. This symmetry is usually obtained through a twist procedure starting from the set of supercharges of an underlying specific supersymmetric theory. In these theories physical states are BRST-like cohomology classes of operators constructed from the fields of the theory which, in some cases, are in correspondence with cohomology cycles of the underlying space-time manifold. The twisted theories have Lagrangians which depend on the background metric however, they can be expressed as an exact BRST-like operator, and consequently the partition function and correlation functions are metric independent. The classical examples of these theories are the twisted $\mathcal{N}=2$ Yang-Mills theory weakly coupled in four dimensions [1] and [2] in the strong coupling limit. The underlying topological invariants are the Donaldson and Seiberg-Witten invariants of four manifolds respectively. Another important example is the topological sigma model [3] giving rise to the Gromow-Witten invariants of the underlying target space.

Another kind of topological field theories are those of the Schwarz type [4]. Their Lagrangian is independent on the background metric at the tree level but the quantization procedure requires the introduction of a Riemannian metric in the computation of the one-loop partition function (the Reidemeister-Ray-Singer analytic torsion [5]) turns out to be metric independent (up on the choice of a framing), giving a topological invariant. An example of topological theories of this type is the Chern-Simons gauge theory, giving rise to link invariants [6]. In this paper Witten found the Jones polynomial invariants of framed knots and links in terms of correlation functions of products of oriented Wilson lines. In the non-perturbative regime $(k \rightarrow \infty)$ it was found that the theory describes the Jones polynomial, while the perturbative one $(k \rightarrow 0)$ is better written in terms of Vassilev polynomials. (Some reviews can be found in Refs. [7, 8, 9]).

There are also inter-relations among the different types of topological field theories. One of them is the topological sigma model on the target space $T^{*} M$ being an hyperKahler manifold, and $M$ an oriented three-manifold. The effective theory on $M$ leads to a Chern-Simons-like theory giving rise to perturbative link invariants known as the Rozansky-Witten invariants [10]. There are new topological invariants coming from the mixture of different topological field theories. Recent reviews on these topics can be found in Refs. [11, 12].

Another class of theories of the Schwarz type are the $B F$ theories associated with higher dimensional generalizations of the Chern-Simons gauge theories and in general of higher order anti-symmetric tensor fields [13, 14]. In these theories the link invariants in higher dimension can be realized through the correlation functions of certain observables of a suitable $B F$ theory [15, 14]. In the present paper we will follow [15] in order to extend this work to the case when there exists a multi-vector field determined by a flow on the underlying space-time manifold.

On the other side it is very well known that topological and geometrical methods

play a crucial role in the theory of dynamical systems. Orbits in non-singular flows can be associated with homology cycles (of dimension one). These cycles are known as 
asymptotic cycles and they were introduced some years ago by Schwartzman [16] for the case of cycles of dimension one, i.e., they are elements of $H_{1}(M, \mathbb{R})$, with $M$ being foliated. Such a "diffuse" cycles are defined as the average homological placement of the periodic orbits of the flow with respect to some invariant probability measures. These asymptotic cycles are genuine homology cycles and the generalization to higher dimensions was done recently in Ref. [17. Such generalization was achieved by using the theory of dynamical systems. In particular, the introduction of flow boxes to define geometric currents [18, 19] was very important. In particular, the results [16] were used to carry over the Jones-Witten polynomial invariants for flows in a dynamical system [20]. In the process Arnold's result [21, 22] of the asymptotic Hopf invariant is obtained (for recent work concerning the asymptotic linking number, see [23] $)^{3}$. Moreover, recently a great deal of work (basically by mathematicians) have been done in classifying dynamical systems by using different invariants from knot theory [20, 25, 26, 27]. For a recent review on the subject see [28].

In the present paper we obtain a generalization of asymptotic linking numbers in higher dimensions. They will constitute new invariants of smooth dynamical systems. One of our main results is the fact that the correlation function of two suitable asymptotic observables defines a higher dimensional generalization of the average asymptotic linking number of a flow that leaves invariant the volume form.

The paper is organized as follows. In Sec. 2 we recall the higher dimensional description of the linking numbers mainly following [15] and [14]. Sec. 3 is devoted to recall the definition of asymptotic cycles and their higher-dimensional generalization. For this we follow Ref. [17]. We give a brief overview of the Arnold's work concerning the asymptotic Gauss linking number. Sections 4, 5 and 6 constitute the main part of our work. In Sec. 4 it is introduced the higher-dimensional linking number for one and two flows in the abelian BF theory (without a cosmological term). The generalized linking number with a non-abelian gauge connection is discussed in the context of one and two flows. In Sec. 5 the asymptotic Jones-Witten invariants discussed at [20] are extended to any odd $(n=2 p+1)$ dimension. Sec. 6 is devoted to argue on a possible relation of asymptotic invariants and string theory and at the same time it would constitutes a physical interpretation on the correlators of observables of RR fields. Finally Sec. 7 contains some concluding remarks.

\section{Overview of Linking Numbers in Quantum Field Theory}

In the present section we overview the higher dimensional linking number. We will follows closely the work of Horowitz and Srednicki [15]. The notation and conventions are taken also from this reference.

\footnotetext{
${ }^{3}$ It is interesting to note that the Arnold's invariant coincides precisely with the helicity, which is a topological quantity arising in some physical and astrophysical processes and that remains invariant under the evolution of the system. It is precisely the behavior of the magnetic field in the plasma inside planets or stars which is described by the helicity [24, 21, 22].
} 
Let $M$ be a $n$-dimensional, closed (compact and without boundary) oriented manifold, let $U$ and $V$ be nonintersecting oriented submanifolds of dimension $p$ and $p^{\prime}=$ $n-p-1$ respectively. Assume that $U$ and $V$ are homologically trivial surfaces, that means they are boundaries of higher dimensional surfaces. Then let $V$ be the boundary of $W$ i.e., $V=\partial W$. We will consider also that $U$ and $W$ are going to intersect only in a finite number of points denoted by $p_{i}$. We define $\operatorname{sign}\left(p_{i}\right)=1$ if the orientation agrees with the orientation on $M$ and $\operatorname{sign}\left(p_{i}\right)=-1$ otherwise. Then the linking number is defined as

$$
L(U, V)=\sum_{i} \operatorname{sign}\left(p_{i}\right)
$$

If $U$ and $V$ are non-intersecting circles $\left(p=p^{\prime}=1\right)$ embedded in $\mathbb{R}^{3}$, another way of calculating the linking number is due Gauss and is given by

$$
L(U, V)=\frac{1}{4 \pi} \int_{U} d x^{i} \int_{V} d y^{j} \varepsilon_{i j k} \partial^{k}|x-y|^{-1} .
$$

It is well known that Chern-Simons (CS) theory is used to calculate links invariants as the Jones polynomial [6] and the Alexander polynomial (see, for instance [12]). In Refs. [13, 14] it was introduced a generalization of the CS functional known as $\mathrm{BF}$ theory which is defined in a compact, oriented, without boundary $n$-dimensional manifold $M$ whose action is expressed as

$$
S_{B F}=\int_{M} B \wedge d C
$$

where $B$ is a $p$-form and $C$ a $p^{\prime}$ form on $M, d$ denotes the exterior derivative acting over forms on $M$. The equations of motion are given by: $d B=0$ and $d C=0$. This action is invariant under diffeomorphisms and the following gauge transformations:

$$
B \rightarrow B+d v, \quad C \rightarrow C+d w,
$$

where $v$ and $w$ are $(p-1)$ and $(n-p-2)$ forms respectively. The moduli space of the theory (the inequivalent gauge field configurations) consist of the elements of the de Rham cohomology groups $H^{p}(M) \times H^{p^{\prime}}(M)$.

The important object to compute is the two-point correlation function of gauge invariant observables $\mathcal{O}_{U}=\int_{U} B$ and $\mathcal{O}_{V}=\int_{V} C$ given by

$$
\left\langle\int_{U} B \cdot \int_{V} C\right\rangle=\frac{\int \mathcal{D} B \mathcal{D} C \int_{U} B \cdot \int_{V} C e^{i S_{B F}}}{\int \mathcal{D} B \mathcal{D} C e^{i S_{B F}}} .
$$

In Ref. [15] it was proved that this is precisely the higher dimensional generalization of the linking number $L(U, V)$ of two homologically trivial cycles $U$ and $V$ given by

$$
\left\langle\int_{U} B \cdot \int_{V} C\right\rangle=i L(U, V) .
$$

If $p$ is odd, $n=2 p+1$ and setting $C=B$, then the action becomes the Chern-Simons functional $S=\int B \wedge d B$. In the procedure to prove (마) we can choose eigenforms satisfying $* d B_{n}=\lambda_{n} B_{n}$ and consequently we get 


$$
\left\langle\int_{U} B \cdot \int_{V} B\right\rangle=i L(U, V)
$$

in this case $U$ and $V$ are $p$-dimensional surfaces.

For the case $M=\mathbb{R}^{n}$ we have that the linking number of $p$ and $p^{\prime}=n-p-1$ dimensional surfaces results [29]

$$
L(U, V)=\frac{\Gamma\left(\frac{n}{2}\right)}{\left[(2 n-4) \pi^{n / 2} p !((n-p-1) !]\right.} \int_{U} d x^{j_{1} \cdots j_{p}} \int_{U} d y^{j_{p+1} \cdots j_{n-1}} \varepsilon_{j_{1} \cdots j_{n-1}}^{j_{n}} \partial_{j_{n}}|x-y|^{2-n}
$$

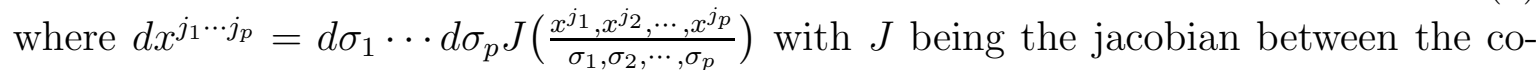
ordinates $x^{\prime} s$ of $\mathbb{R}^{n}$ and the worldvolume coordinates $\sigma^{\prime} s$ of $U$. The case $U=V$ $(x=y)$ is divergent (coincident singularity) and one can have an invariant regularization procedure [6, 30] through the choice of a framing (smooth vector field on $M$ ). The asymptotic invariant that we will introduce in Sec. 4 incorporates automatically this vector field (or multi-vector field in higher dimensions) and consequently contains an invariant regularization to the self-linking number (8) of the flow and the choice of a framing through the choice of a $p$-vector field $\mathbf{X}_{p}=X_{1} \wedge \cdots \wedge X_{p}$ from the $p$-tensor product of the tangent bundle of $M$.

\subsection{Generalized Linking Number}

Let $A$ be a flat connection on a $G$-principal bundle $E$ over $M$ with $G$ a compact connected Lie group. Let us take $\mathcal{B} \in H^{p}(M, E)$ and $\mathcal{C} \in H^{p^{\prime}}(M, E)$ (with $\left.p^{\prime}=n-p-1\right)$ transforming in a non-trivial dual representations of $G$. Thus can take $\mathcal{B}$, for instance, transforming in the fundamental representation $\square$ of $G$, while $\mathcal{C}$ will transform in the anti-fundamental representation $\bar{\square}$. Consider the following action

$$
S=\int_{M} \mathcal{B} \wedge D \mathcal{C}
$$

where $D=d+A$ is the covariant derivative and satisfies $D^{2}=0$. This action is invariant under the infinitesimal gauge transformations $\delta \mathcal{B}=D v$ and $\delta \mathcal{C}=D w$. The equations of motion are: $D \mathcal{B}=0$ and $D \mathcal{C}=0$. The space of solutions is the moduli space given by $H^{p}(M, E) \times H^{p^{\prime}}(M, E)$. Following a similar procedure to the case without the gauge potential (from Ref. [15]) we want to extract a gauge invariant quantity, then we must pick two surfaces $U$ and $V$ and take a point in each one of them and a family of curves (homotopy class) $\gamma$ that join any pair of points $x \in U$ and $y \in V$. This family define a Wilson line, then the correlation function of observables constructed with $\mathcal{B}, \mathcal{C}$ and $A$ defines a generalized linking number $L_{A}(U, C)$ as follows

$$
L_{A}(U, V)=-i\left\langle\int_{U} \mathcal{B}(x) \int_{V} P \exp \left(\int_{x}^{y} A\right) \mathcal{C}(y)\right\rangle .
$$

This quantity transforms as $\bar{\square}_{x} \otimes\left(\bar{\square}_{x}, \square_{y}\right) \otimes \bar{\square}_{y}$ and it is certainty an invariant of the group and consequently gauge invariant. 
This expression is metric independent (because the longitudinal and zero modes do not contribute to the integral). In this case we need that $U$ and $V$ be homologically trivial as in the previous case, but we need one extra condition, because the Wilson line jumps discontinuously for a non-trivial holonomy loop. Thus to ensure that longitudinal modes do not contribute the Wilson lines are continuous in $x$ and $y$. This statement concerning continuity is expressed in the following definition.

A surface $U$ is holonomically trivial if every closed curve on $U$ has trivial holonomy. This condition is related with the homotopy of $U$, if every element of $\pi_{1}(U)$ is homotopically trivial in $M$ then $U$ is holonomically trivial, then the longitudinal and zero modes not contribute to the integral. In addition there exist holonomically trivial surfaces $W$ and $W^{\prime}$ such that $\partial W=V$ and $\partial W^{\prime}=U$ (because $\int_{V} P \exp \left(\int_{x}^{y} A\right) \mathcal{C}^{0}(y)=\int_{W} P \exp \left(\int_{x}^{y} A\right) D \mathcal{C}^{0}(y)$ where $D \mathcal{C}^{0}=0$ and $\mathcal{C}^{0}$ is a zero mode $)$. Thus we obtain the following expression for the generalized linking number

$$
L_{A}(U, V)=\sum_{i} \operatorname{sign}\left(p_{i}\right) \operatorname{Tr} P \exp \left(\oint_{\gamma_{i}} A\right),
$$

with the closed curve $\gamma_{i}$ starting at $p_{i}$, following a curve in $U$ to $u_{0}$, then go around $\gamma_{0}$ to $v_{o}$, and finally a curve go back to $p_{i}$ keeping inside $W$.

\section{Dynamics of Flows and Their Invariants}

In this section we overview briefly some definitions and mathematical results concerning geometry and topology of dynamical systems for future reference. Notation and conventions is taken mainly from Refs. [16, 18, 17, 31. We also overview an application of these results to the computation of the Hopf invariant for the flow.

\subsection{Asymptotic Cycles}

One dimensional asymptotic cycles were introduced by Schwartzman in [16]. In order to define them, we first consider a manifold $M$ with a flow $f_{t}$ and take a fixed set of regular curves $\left\{\gamma_{p, q}\right\}$ joining any pair of points $p$ and $q$ of $M$. For a given point $p$ and for any $t>0$, we define the integral-singular 1-cycle $\widetilde{\Gamma}_{t, p}=\left[p, f_{t}(p)\right] \cup \gamma_{f_{t}(p), p}$, where $\left[p, f_{t}(p)\right]$ is the oriented arc of trajectory going from $p$ to $f_{t}(p)$. If we define $\Gamma_{p, t}=\frac{1}{t} \tilde{\Gamma}_{t, p}$ one can prove [16] that the $\lim _{t \rightarrow \infty}\left[\Gamma_{t, p}\right]=\left[\Gamma_{p}\right]$ exists and belongs to $H_{1}(M, \mathbb{R})$. For every quasi-regular point $p$ of $M$, it is independent of the metric and the connected curves $\gamma_{p, q}$ in $M$.

Now let $\mu$ be a measure on $M$ invariant under the flow and $\omega$ a closed 1 -form in the de Rham cohomology group $H_{d R}^{1}(M, \mathbb{R})$. Define the linear functional

$$
\Psi_{\mu}: H_{d R}^{1}(M, \mathbb{R}) \rightarrow \mathbb{R}, \quad \Psi_{\mu}([\omega])=\int_{M} \omega(X) \mu
$$

where $X$ is the vector field generated by the flow and $\omega$ will depend only on the

cohomology class $[\omega] . \quad \Psi_{\mu}$ as a current [31] on the de Rham cohomology defines a 
homology cycle which can be regarded as a winding cycle for each invariant probability ergodic measure $\mu$ of $M$.

For a higher dimensional generalization of asymptotic cycles we consider first $\mathcal{S}$ to be a closed subset of a $n$-dimensional manifold $M$. A partial foliation of dimension $p$ consists of a family of $L_{\alpha}$ (whose dimension is such that $\mathcal{S}=\cup_{\alpha} L_{\alpha}$. A collection of flow boxes on $M$ is a collection of closed disks $\mathbf{D}^{p} \times \mathbf{D}^{n-p}$ (horizontal and vertical disk respectively), whose interior cover $M$ and intersect each $L_{\alpha}$ in a collection of horizontal disks $\left\{\mathbf{D}^{p} \times\{y\}\right\}$. We consider that the disks are smoothly embedded, such that the tangent planes vary continuously on the flow boxes.

A $(n-p)$-dimensional submanifold $T$ of $M$ is said to be transversal if it is transversal each $L_{\alpha}$. We say $T$ will be small if it is contained in a single flow box. Then a transversal measure $\mu_{T}$ provides to each small transversal submanifold $T$ with a measure. We will assume that each measure is supported on the transversal, intersecting support of the current which is invariant under the flow. Then we define a geometrical current as the triple $\left(L_{\alpha}, \mu_{T}, \nu\right)$, with the entries being objects defined as above. The geometric current assigns to each point in the support $\mathcal{S}$ an orientation $\nu$ of $L_{\alpha}$, through such point.

Suppose that $M$ is covered by a system of flow boxes $\left\{\left(\mathbf{D}^{p} \times \mathbf{D}^{n-p}\right)_{i}\right\}$ endowed with partitions of unity. Then, every $p$-form $\omega$ can be decomposed into a finite sum $\omega=\sum_{i} \omega_{i}$, where each $\omega_{i}$ has his own support in the $i$-th flow box. Now we can integrate out every $\omega_{i}$ over each horizontal disk $\left(\mathbf{D}^{p} \times\{y\}\right)_{i}$ and obtain a continuous function $f_{i}$ over $\left(\mathbf{D}^{n-p}\right)_{i}$. Thus we can take the average of this function using the transversal measures $\mu$ to obtain a number. Therefore we define a current given by

$$
\left\langle\left(L_{\alpha}, \mu, \nu\right), \omega\right\rangle=\sum_{i} \int_{\left(\mathbf{D}^{n-p}\right)_{i}} \mu_{T}(d y)\left(\int_{\left(\mathbf{D}^{p} \times\{y\}\right)_{i}} \omega_{i}\right) .
$$

This current is closed in the sense of de Rham [31], i.e., if $\omega=d \phi$, where $\phi$ has compact support, then $\left\langle\left(L_{\alpha}, \mu, \nu\right), d \phi\right\rangle=0$, since we can write $\phi=\sum_{i} \phi_{i}$. Ruelle and Sullivan [18] show that this current determines precisely an element of the $p$-th cohomology group. In this case, if $\mu$ is invariant it does not depend of the choice of system of flow boxes that was used. Recall that any $(n-p)$-form $\rho$ on $M$ determines a $p$-dimensional current by Poincaré duality $\langle\rho, \omega\rangle=\int_{M} \omega \wedge \rho$.

Now consider an example of geometrical current. Let $\mu$ be an invariant volume form and $\mathbf{X}_{p}$ is a $p$-vector field nowhere vanishing on $M$, this defines a transversal measure defined by a $(n-p)$-form $\eta=i_{\mathbf{X}_{p}}(\mu)$. The de Rham current is

$$
\begin{aligned}
C_{\mu, \mathbf{X}_{p}}(\beta) & =\sum_{i} \int_{\mathbf{D}_{i}^{n-p}} \eta \int_{\mathbf{D}_{i}^{p} \times\{y\}} \beta \\
& =\int_{M} i_{\mathbf{X}_{p}}(\mu) \wedge \beta .
\end{aligned}
$$

This determines a closed current when $\mu$ is invariant under the flow described by $\mathbf{X}_{p}$, here $\beta$ is a $p$-form.

Now we will give some useful results concerning the existence of higher dimensional asymptotic cycles (for more details see [17]). Consider the action of a connected Lie 
group $L$ on a smooth compact oriented manifold $M$, whose orbits are tangent to the orbits of dimension $p$. A quantifier is a continuous field of $p$-vectors on $M$ everywhere tangent to the orbits and invariant under the action of $L$. A quantifier is said to be positive if it is nowhere vanishing and determines the orientation of the tangent space. A preferred action is an oriented action of a connected Lie group $L$ such that for any $x \in M$ the isotropy group $D_{x}$ of $x$ is a normal subgroup of $L$ and $L / D_{x}$ is unimodular.

It was proved in [17] that a preferred action possesses a positive quantifier. Given a positive quantifier we define a $1-1$ correspondence between a finite invariant measure $\mu$ and a transversal invariant measure. An important result which will be used in the next sections is a theorem that states as follows. If $\mathbf{X}_{p}$ is a positive definite quantifier and $\mu$ is an invariant measure described by a $n$-form, then $i_{\mathbf{X}_{p}}(\mu)$ is a closed $(n-p)$ form and the asymptotic cycle $A_{\mu}$ can be obtained by Poincaré duality of an element of $H^{n-p}(M, \mathbb{R})$ determined by $i_{\mathbf{X}_{p}}(\mu)$.

If $A_{\mu}$ is an asymptotic cycle i.e. is an element of $H_{p}(M, \mathbb{R})$, the theorem give us an explicit form to construct asymptotic cycles if the above conditions are satisfied. This is not the only way to specify a foliation, in [19] Sullivan defines structures of $p$-cones and operators acting over vectors on these cones.

\subsection{Asymptotic Hopf Invariant for a Flow}

Using the idea of asymptotic cycle we define asymptotic average linking number as in Ref. [21]. Let $M$ be a closed and orientable 3-manifold with volume form (invariant measure) $\mu$, which we will assume that is normalized as $\int_{M} \mu=1$. Consider a vector field $X$ that induces a flow $f_{t}$, which satisfies $\mathcal{L}_{X} \mu=0$ (divergence-free vector field) which is homologically trivial i.e., there exist a 1-form $\alpha$ such that $d \alpha=i_{X}(\mu)$. For a closed 1-form the winding cycle is zero for a homologically trivial vector field

$$
\begin{gathered}
\Psi_{\mu}=\int_{M} \omega(X) \mu=\int_{M} \omega \wedge i_{X}(\mu) \\
=\int_{M} d(\omega \wedge \alpha)=0 .
\end{gathered}
$$

Under this situation the asymptotic linking number exists and is well defined [21, 22, 23 ,

$$
L\left(\Gamma_{x_{1}}, \Gamma_{x_{2}}\right)=\lim _{T_{1}, T_{2} \rightarrow \infty} \frac{L\left(\widetilde{\Gamma}_{T_{1}, x_{1}}, \widetilde{\Gamma}_{T_{2}, x_{2}}\right)}{T_{1} T_{2}}
$$

where $\widetilde{\Gamma}_{t, x_{i}}=\left[x_{i}, f_{t}\left(x_{i}\right)\right] \cup \gamma_{f_{t}\left(x_{i}\right), x_{i}}$, with $\left[x_{i}, f_{t}\left(x_{i}\right)\right]$ being the oriented arc of trajectory from $x_{i}$ to $f_{t}\left(x_{i}\right)$ and $\left\{\gamma_{f_{t}\left(x_{i}\right), x_{i}}\right\}$ is a set of regular curves that join $f_{t}\left(x_{i}\right)$ and $x_{i}$ with $i=1,2$.

The mean value of the asymptotic linking number of a pair of trajectories (average Hopf invariant) of a homologically trivial vector field $X$ on $M$ is given by

$$
L(X)=\int_{M \times M} L\left(\Gamma_{x_{1}}, \Gamma_{x_{2}}\right) d v\left(x_{1}\right) d v\left(x_{2}\right)
$$


Finally Arnold proved [21, 22] that this number is equal to

$$
L(X)=\int_{M} \alpha \wedge d \alpha
$$

where $d \alpha=i_{X}(\mu)$. The explicit form for $L(X)$ is given by

$$
L(X)=\int_{M \times M} \frac{\varepsilon_{i j k} X^{i}\left(x_{1}\right) X^{j}\left(x_{2}\right)\left(x_{1}-x_{2}\right)^{k}}{\left|x_{1}-x^{2}\right|^{3}} d v\left(x_{1}\right) d v\left(x_{2}\right) .
$$

\section{Asymptotic Linking Numbers in Higher Dimen- sions}

In this section we generalize some of the material revisited in Sec. 2 to higher dimensions. In order to do that we will use the definition of asymptotic cycles in higher dimensions from [17]. Our main motivation is the extension of the ideas of dynamical systems to string theory.

\subsection{Average Asymptotic Linking Number}

Now we proceed to give the definition of the asymptotic linking number in higher dimensions using the standard $B F$ action without cosmological constant term (3) given in section 2 .

Let us consider $M$ to be a $n$-dimensional manifold such that $H_{p}(M, \mathbb{R})=0$. $M$ is also equipped with an invariant flow volume form $\mu$ and a divergence-free $p$-vector field $\mathbf{X}_{p}=X_{1} \wedge \cdots \wedge X_{p}$ i.e. $\mathcal{L}_{\mathbf{X}_{p}} \mu=0$ (see [32, 33] for notation, conventions and properties regarding multi-vector fields). Here each $X_{i}$ with $i=1, \cdots, p$ is a vector field (1-vector field). For a $p$-vector field the Lie derivative is defined as: $\mathcal{L}_{\mathbf{X}_{p}}=\left[i_{\mathbf{X}_{p}}, d\right]$, where $i_{\mathbf{X}_{p}}$ is defined by $i_{\mathbf{X}_{p}}=i_{X_{1} \wedge \cdots \wedge X_{p}}=i_{X_{1}} \circ \cdots \circ i_{X_{p}}$ is the contraction, notice that all $i_{X_{i}}$ 's commutes among themselves). If $N=\partial W$ is a null homologous $\left(p^{\prime}=n-p-1\right)$ dimensional submanifold of $M, N$ is boundary of some $(n-p)$-dimensional manifold.

Motivated by [16, 17, 18, 20] we define the following asymptotic observable $\widetilde{O}_{\mathbf{x}_{p}}$ as a current 31]

$$
\widetilde{O}_{\mathbf{X}_{p}}=\int_{M} i_{\mathbf{X}_{p}}(B) \mu_{\mathbf{X}_{p}}\left(p_{1}\right)
$$

Then the two-point correlation function is consequently

$$
\begin{aligned}
\left\langle\widetilde{O}_{\mathbf{X}_{p}}\left(p_{1}\right) \cdot O_{N}\left(p_{2}\right)\right\rangle & =\left\langle\int_{M} i_{\mathbf{X}_{p}}(B) d \mu\left(p_{1}\right) \cdot \int_{N} C\left(p_{2}\right)\right\rangle \\
& =\frac{\int \mathcal{D} B \mathcal{D} C \exp \left[i \int_{M} B \wedge d C\right] \int_{M} i_{\mathbf{X}_{p}}(B) \mu\left(p_{1}\right) \int_{N} C\left(p_{2}\right)}{\int \mathcal{D} B \mathcal{D} C \exp \left[i \int_{M} B \wedge d C\right]} .
\end{aligned}
$$

These observables are invariant under the gauge transformations (4). In the present case it can be written as

$$
\int_{M} i_{\mathbf{X}_{p}}\left(B^{\prime}\right) \mu\left(p_{1}\right)=\int_{M} i_{\mathbf{X}_{p}}(B) \mu_{\mathbf{X}_{p}}\left(p_{1}\right)+\int_{M} i_{\mathbf{X}_{p}}(d v) \mu_{\mathbf{X}_{p}}\left(p_{1}\right),
$$


where $v$ is a $(p-1)$-form. The second term vanishes due the theorem $2 \mathrm{~A}$ in [17], which states that the current is closed if and only if $\mu$ is an invariant measure. The second observable $\int_{N} C$ is clearly gauge invariant.

In order to compute the rhs expression of (21), as in the case without flows [15], we again split the forms using the Hodge decomposition theorem. If $B$ is a $p$-form we can write down it uniquely as: $B=B^{0}+d \phi+\delta \xi$, where $B^{0}$ is the harmonic part, $d \phi$ and $\delta \xi$ are the longitudinal and transversal parts of $B$ respectively, $d$ is the usual exterior derivative and $\delta=(-1)^{p n+n+1} * d *$ the adjoint. Here $\phi$ is a $(p-1)$-form and $\xi$ is a $(p+1)$-form, $*$ is the Hodge operator on $M$ such that $*^{2}=(-1)^{p(n-p)}$.

To evaluate this integral, we split the measure in the following way with the aid of the Hodge theorem: $\mathcal{D} B=\mathcal{D} B^{T} \mathcal{D} B^{L} \mathcal{D} B^{0}$ and similarly for $\mathcal{D} C$. Only the transverse part of the $B F$ action and observables contributes, i.e. $\widetilde{O}_{\mathbf{X}_{p}}=\int_{M} i_{\mathbf{X}_{p}}\left(B^{T}\right) \mu$ and $O_{N}=\int_{N} C^{T}$ therefore we integrate out all longitudinal and harmonic modes leaving only the transverse ones $\mathcal{D} B^{T} \mathcal{D} C^{T}$.

Consider $\lambda^{2} \neq 0$ to be an eigenvalue of the laplacian $\Delta_{p}=\delta d+d \delta$ let $\Lambda_{p}$ the space of $p$ eigenforms $\left(\Delta B=\lambda^{2} B\right)$. This space is partitioned in the transverse $\Lambda_{p}^{T}$ and longitudinal $\Lambda_{p}^{L}$ parts. Following to [15] we have an isomorphism between $\Lambda_{p}$ and $\Lambda_{n-p}$ due to the Hodge dual maps $p$-forms with $\lambda^{2}$ to $(n-p)$-forms with the same eigenvalue. Also we have that the codifferential is a mapping from $\Lambda_{p}^{L}$ to $\Lambda_{p-1}^{T}$ and the differential $d$ maps from $\Lambda_{p}^{T}$ to $\Lambda_{p+1}^{L}$. Thus we can define the following map $\lambda^{-1} * d: \Lambda_{p}^{T} \rightarrow \Lambda_{n-p-1}^{T}$, which define an isomorphism.

Let $\left\{B_{j}\right\}$ and $\left\{C_{j}\right\}$ be basis of normalized transverse eigenforms which satisfies $\left\langle B_{j} \mid B_{k}\right\rangle=\int_{M} B_{j} \wedge * B_{k}=\delta_{j k}$. If one takes the following choice for $C_{j}=(-1)^{n-p-1} \lambda_{j}^{-1} *$ $d B_{j}$, then the $C_{j}$ are also orthonormal. Let us take $B$ and $C$ in their respective expansion of the basis $B=\sum_{j} b_{j} B_{j}$ and $C=\sum_{j} c_{j} C_{j}$, then the action can be written as: $S_{B F}=\langle B \mid C\rangle=\sum_{j} \lambda_{j} b_{j} c_{j}$ due to the normalization of $B_{j}$ and $C_{j}$. The transverse measure of the path integral takes the following form $\mathcal{D} B^{T} \mathcal{D} C^{T}=\prod_{j} d b_{j} \prod_{k} d c_{k}$. Substituting and integrating (21) this yields

$$
\begin{gathered}
H\left(\mathbf{X}_{p}, N\right):=\frac{1}{i}\left\langle\widetilde{O}_{\mathbf{X}_{p}}\left(p_{1}\right) \cdot O_{N}\left(p_{2}\right)\right\rangle \\
=\left.\sum_{j} \lambda_{j}^{-1} \int_{M} i_{\mathbf{X}_{p}}\left(B_{j}\right)\right|_{p_{1}} \mu\left(p_{1}\right) \cdot \int_{N} C_{j}\left(p_{2}\right) .
\end{gathered}
$$

Using the properties of $i_{X_{1} \wedge \cdots \wedge X_{p}}$ it is easy to see that

$$
i_{X_{1} \wedge \cdots \wedge X_{p}} B_{j} \wedge \mu-(-1)^{\frac{p}{2}(3+p)} B_{j} \wedge i_{X_{p} \wedge \cdots \wedge X_{1}} \mu=0 .
$$

Substituting the last equality in (23), we obtain

$$
H\left(\mathbf{X}_{p}, N\right)=\left.(-1)^{\frac{p}{2}(3+p)} \sum_{j} \lambda_{j}^{-1} \int_{M} B_{j}\right|_{p_{1}} \wedge i_{\mathbf{X}} \mu\left(p_{1}\right) \cdot \int_{N} C_{j}\left(p_{2}\right) .
$$

If $\mathbf{X}_{p}$ is a divergence-free $p$-vector field, this implies that $\eta=i_{\mathbf{X}_{p}} \mu$ is closed. Since $H_{p}(M, \mathbb{R})$ is trivial then by Poincaré duality there exists $\alpha \in \Omega^{n-p-1}(M)$ such that $\eta=d \alpha^{\prime}$, where $\alpha^{\prime}:=(-1)^{\frac{p}{2}(3+p)} \alpha$. Then $N$ is null-homologous i.e. $N=\partial W$, where $W$ is a $(n-p)$-manifold we get 


$$
\begin{aligned}
H\left(\mathbf{X}_{p}, N\right) & =\left.\sum_{j} \lambda_{j}^{-1} \int_{M} B_{j}\right|_{p_{1}} \wedge \eta\left(p_{1}\right) \cdot \int_{N} C_{j}\left(p_{2}\right) \\
& =\left.\sum_{j} \lambda_{j}^{-1} \int_{M} B_{j}\right|_{p_{1}} \wedge \eta\left(p_{1}\right) \cdot \int_{W} d C_{j}\left(p_{2}\right) .
\end{aligned}
$$

Using $d C_{j}=\lambda_{j} * B_{j}$ we obtain the following expression

$$
H\left(\mathbf{X}_{p}, N\right)=\sum_{j} \int_{M} \int_{W} B_{j}\left(p_{1}\right) * B_{j}\left(p_{2}\right) \wedge \eta\left(p_{1}\right) .
$$

Using the completeness relation for the eigenforms i.e.

$\sum_{j} B_{j}^{T}\left(p_{1}\right) * B_{j}^{T}\left(p_{2}\right)+\sum_{j} B_{j}^{L}\left(p_{1}\right) * B_{j}^{L}\left(p_{2}\right)+\sum_{j} B_{j}^{0}\left(p_{1}\right) * B_{j}^{0}\left(p_{2}\right)=\delta\left(p_{1}, p_{2}\right) v\left(p_{1}\right) \cdot v\left(p_{2}\right)$,

where $v\left(p_{1}\right)$ and $v\left(p_{2}\right)$ are $p$ and $n-p$ "volume" forms in a $p$ foliation of $M$. Since the longitudinal and harmonic forms do not contribute to the path integral we can include them and integrate over $M$, we have the following result

$$
\begin{gathered}
H\left(\mathbf{X}_{p}, N\right)=\int_{M} \int_{W} \delta\left(p_{1}, p_{2}\right) v\left(p_{1}\right) \cdot v\left(p_{2}\right) \wedge \eta\left(p_{1}\right) \\
=\int_{W} \eta\left(p_{1}\right) \\
=\int_{N} \alpha^{\prime} .
\end{gathered}
$$

This is precisely a Hopf type integral. It is immediate to note that for the case of $p=1$ we get the result $\int_{N} \alpha$ by Kotschick and Vogel [25]. For this case $H_{1}(M, \mathbb{R})$ is trivial, $X$ is a divergence-free vector field, $\alpha$ is a $(n-2)$-form, which satisfies $i_{X}(\mu)=d \alpha$.

\subsection{Asymptotic Intersection of Two Flows}

In this subsection we will calculate a correlation function of two asymptotic observables. This leaves us to define the asymptotic linking number between two flows of dimension $p$ and $p^{\prime}$. After that we will calculate the asymptotic self-intersection and finally extract the Hopf invariant in higher dimensions.

Let $\widetilde{O}_{\mathbf{x}_{p}}$ and $\widetilde{O}_{\mathbf{Y}_{p^{\prime}}}$ be two asymptotic observables defined as in Eq. (20). We will consider that each observable has it own flow invariant measure $\mu_{\mathbf{x}_{p}}$ and $\mu_{\mathbf{Y}_{p^{\prime}}}$, where $\mathbf{X}_{p}=X_{1} \wedge \ldots \wedge X_{p}$ and $\mathbf{Y}_{p^{\prime}}=Y_{1} \wedge \ldots \wedge Y_{p^{\prime}}$. Suppose $M$ has trivial $p$-th and $(p+1)$-th homology groups then the two point correlation function to calculate is the following:

$$
\begin{aligned}
& \left\langle\widetilde{O}_{\mathbf{x}_{p}}\left(p_{1}\right) \cdot \widetilde{O}_{\mathbf{Y}_{p^{\prime}}}\left(p_{2}\right)\right\rangle=\frac{\int \mathcal{D} B \mathcal{D} C \exp \left[i \int_{M} B \wedge d C\right] \widetilde{O}_{\mathbf{x}_{p}}\left(p_{1}\right) \cdot \widetilde{O}_{\mathbf{Y}_{p^{\prime}}}\left(p_{2}\right)}{\int \mathcal{D} B \mathcal{D} C \exp \left[i \int_{M} B \wedge d C\right]} \\
& =\frac{\int \mathcal{D} B \mathcal{D} C \exp \left[i \int_{M} B \wedge d C\right] \int_{M} i_{\mathbf{x}_{p}}(B) \mu_{\mathbf{x}_{p}}\left(p_{1}\right) \cdot \int_{M} i_{\mathbf{Y}_{p^{\prime}}}(C) \mu_{\mathbf{Y}_{p^{\prime}}}\left(p_{2}\right)}{\int \mathcal{D} B \mathcal{D} C \exp \left[i \int_{M} B \wedge d C\right]} .
\end{aligned}
$$


Remember that to the path integral only contributes the transversal part since the longitudinal modes and the harmonic decouples from the transverse ones and can be directly integrated out (29) and it reduces to

$$
\begin{gathered}
\left\langle\widetilde{O}_{\mathbf{X}_{p}}\left(p_{1}\right) \cdot \widetilde{O}_{\mathbf{Y}_{p^{\prime}}}\left(p_{2}\right)\right\rangle \\
=\left.\left.i \sum_{j} \lambda_{j}^{-1} \int_{M} i_{\mathbf{X}_{p}}(B)\right|_{p_{1}} \mu_{\mathbf{X}_{p}}\left(p_{1}\right) \cdot \int_{M} i_{\mathbf{Y}_{p^{\prime}}}(C)\right|_{p_{2}} \mu_{\mathbf{Y}_{p^{\prime}}}\left(p_{2}\right) .
\end{gathered}
$$

Dividing by $i$ we define the asymptotic linking number $H\left(\mathbf{X}_{p}, \mathbf{Y}_{p^{\prime}}\right)$ of $\mathbf{X}_{p}$ and $\mathbf{Y}_{p^{\prime}}$ as follows

$$
\begin{gathered}
H\left(\mathbf{X}_{p}, \mathbf{Y}_{p^{\prime}}\right):=\frac{1}{i}\left\langle\widetilde{O}_{\mathbf{X}_{p}}\left(p_{1}\right) \cdot \widetilde{O}_{\mathbf{Y}_{p^{\prime}}}\left(p_{2}\right)\right\rangle \\
=\left.\left.\sum_{j} \lambda_{j}^{-1} \int_{M} B_{j}\right|_{p_{1}} \wedge \eta_{1}\left(p_{1}\right) \int_{M} C_{j}\right|_{p_{2}} \wedge \eta_{2}\left(p_{2}\right),
\end{gathered}
$$

where we apply (24) to the two integrals. We also use the facts that the fields are divergence-free to prove that $\eta_{1}=i_{X_{1} \wedge \cdots \wedge X_{p}}\left(\mu_{\mathbf{X}_{p}}\right)$ and $\eta_{2}=i_{Y_{1} \wedge \cdots \wedge Y_{p^{\prime}}}\left(\mu_{\mathbf{Y}_{p^{\prime}}}\right)$ are closed and providing our assumption that $H_{p}(M, \mathbb{R})=H_{p+1}(M, \mathbb{R})=0$ they are exact i.e., $\eta_{1}=d \alpha_{1}^{\prime}$ and $\eta_{2}=d \alpha_{2}^{\prime}$, where $\alpha_{1}^{\prime}=(-1)^{\frac{p}{2}(3+p)} \alpha_{1}$ and $\alpha_{2}^{\prime}=(-1)^{\frac{p^{\prime}}{2}\left(3+p^{\prime}\right)} \alpha_{2}$ with $\alpha_{1} \in \Lambda^{p}(M)$ and $\alpha_{2} \in \Lambda^{p^{\prime}}(M)$.

Integrating by parts the second integral, using again the fact that $d C_{j}=\lambda_{j} * B_{j}$, and summing over all the states (including the longitudinal and harmonic forms) we get

$$
\begin{aligned}
H\left(\mathbf{X}_{p}, \mathbf{Y}_{p^{\prime}}\right)= & \int_{M} \int_{M} \delta\left(p_{1}, p_{2}\right) \wedge \eta_{1}\left(p_{1}\right) \alpha_{2}\left(p_{2}\right) \\
& =\int_{M} d \alpha_{1}^{\prime} \wedge \alpha_{2}^{\prime} .
\end{aligned}
$$

Thus we can think of this expression as a Hopf like invariant or as the asymptotic intersection associated to the vector fields $\mathbf{X}_{p}$ and $\mathbf{Y}_{p^{\prime}}$. Let us see some considerations.

As an example we would like to compute the asymptotic self-linking number of a divergence-free $p$-vector field $\mathbf{X}_{p}=X_{1} \wedge \cdots \wedge X_{p}$ i.e. we want $H\left(\mathbf{X}_{p}, \mathbf{X}_{p}\right)$ with $\mathbf{X}_{p}=\mathbf{Y}_{p^{\prime}=p}$. If $M$ is of dimension $n=2 p+1$ where for $p \geq 1$ by dualities between homology and cohomology we relax the condition only to assume $p$-th homology group will be zero. From (32) then the expectation value is:

$$
H\left(\mathbf{X}_{p}, \mathbf{X}_{p}\right)=\int_{M} \alpha \wedge d \alpha
$$

where $\alpha$ is a $(p-1)$-form given by the equation $i_{\mathbf{X}_{p}}(\mu)=d \alpha$. This expression is exactly the Hopf invariant (or helicity) in dimension greater than three [34] or the higher dimensional helicity of a $p$-vector field $\mathbf{X}_{p}$ (it can be associated to a $p$-foliation since the observables defines $p$-currents on $M$ ) equipped with an invariant measure $\mu$, associated to a $p$-foliation of $M$. The involved $p$-vector field $\mathbf{X}_{p}$ represents a choice 
of a framing on the tangent bundle of $M_{n}$ and consequently (33) does not have the coincidence singularity and it is perfectly regular.

Clearly for the case when we take $n=3$ and $p=1$, we have the classical result by Arnold, the asymptotic Hopf invariant (or helicity) of a incompressible flow on a 3-manifold [21].

\subsection{Generalized Linking Numbers for Flows}

Now we want to calculate the asymptotic generalized linking number of a $p$-vector field. In order to do this we recall the asymptotic observable defined as in Eq. (20).

\section{One Flow}

Now we consider the intersection of a flow $\mathbf{X}_{p}$ with a null homologous submanifold $N=\partial W$ in $M$ of dimension $p^{\prime}$. Similarly we assume $H_{p}(M, E)$ is trivial where $E$ is a trivial gauge bundle of with $S U(N)$ structure group. Now we define the generalized asymptotic linking number as the following correlation function:

$$
H_{A}\left(\mathbf{X}_{p}, N\right)=\frac{1}{i}\left\langle\int_{M} i_{\mathbf{X}_{p}}(\mathcal{B}) \mu\left(p_{1}\right) \cdot \int_{N} \mathrm{P} \exp \left(\int_{p_{1}}^{p_{2}} A\right) \mathcal{C}\left(p_{2}\right)\right\rangle,
$$

where $\mathcal{B} \in \square$ and $\mathcal{C} \in \bar{\square}, A \in \mathbf{a d j}=(\square, \bar{\square})$. The observable will be gauge invariant and the correlation function will be a scalar. The expectation value is invariant since the currents are invariant under the gauge transformations, provided that $\gamma_{0}$ is a curve that join $p_{1}$ and $p_{2}$. In this case also the transverse modes decouples from the longitudinal and the transverse ones and they factorizes in the path integral in such a way that they can be integrated out. We have

$$
H_{A}\left(\mathbf{X}_{p}, N\right)=\left.\sum_{j} \lambda_{j}^{-1} \int_{M} i_{\mathbf{X}_{p}}\left(\mathcal{B}_{j}\right)\right|_{p_{1}} \mu\left(p_{1}\right) \cdot \int_{N} \mathrm{P} \exp \left(\int_{p_{1}}^{p_{2}} A\right) \mathcal{C}_{j}\left(p_{2}\right)
$$

or

$$
H_{A}\left(\mathbf{X}_{p}, N\right)=\left.\sum_{j} \lambda_{j}^{-1} \int_{M} \mathcal{B}_{j}\right|_{p_{1}} \wedge \eta\left(p_{1}\right) \cdot \int_{N} \mathrm{P} \exp \left(\int_{p_{1}}^{p_{2}} A\right) \mathcal{C}_{j}\left(p_{2}\right) .
$$

Since $N$ is null homologous using Stokes theorem and taking $D \mathcal{C}_{j}=\lambda_{j} * \mathcal{B}_{j}$, we obtain

$$
H_{A}\left(\mathbf{X}_{p}, N\right)=\left.\sum_{j} \int_{M} \mathcal{B}_{j}\right|_{p_{1}} \wedge \eta\left(p_{1}\right) \cdot \int_{W} \mathrm{P} \exp \left(\int_{p_{1}}^{p_{2}} A\right) * \mathcal{B}_{j}\left(p_{2}\right)
$$

in the last equation we have use the fact that $A$ is flat (i.e. $F_{A}=d A+A \wedge A=0$ ). In order to proceed the computation we incorporate the spurious modes, this yields

$$
H_{A}\left(\mathbf{X}_{p}, N\right)=\int_{M} \int_{W} \delta\left(p_{1}, p_{2}\right) v\left(p_{1}\right) \cdot v\left(p_{2}\right) \cdot \operatorname{Tr} \mathrm{P} \exp \left(\int_{p_{1}}^{p_{2}} A\right) \eta\left(p_{1}\right) .
$$

Integrating over $p_{1}$ this equation reduces to 


$$
H_{A}\left(\mathbf{X}_{p}, N\right)=\int_{W} \operatorname{Tr} \mathrm{P} \exp \left(\oint_{\gamma} A\right) \eta
$$

where $\gamma$ is build as follows: take a curve from $p_{2}$ in $W$ to a point $u$ in the Poincaré dual of $\eta$. Then use the curve $\gamma_{0}$ from $u$ to $v$ in $V$ and finally take a curve from $u$ to $p_{2}$ that is contained in $W$.

\section{Two Flows}

Now consider two divergence-free vector fields and $H_{p+1}(M, \mathbb{R})=H_{p^{\prime}+1}(M, \mathbb{R})=0$. The first one a $p$-vector field $\mathbf{X}_{p}$ and $p^{\prime}$-vector field $\mathbf{Y}_{p^{\prime}}$. Every field has his own flow invariant measure, let say $\mu_{\mathbf{X}_{p}}$ and $\mu_{\mathbf{Y}_{p^{\prime}}}$ respectively. We extend (29) and then the generalized asymptotic linking number $H_{A}\left(\mathbf{X}_{p}, \mathbf{Y}_{p^{\prime}}\right)$ as follows

$$
H_{A}\left(\mathbf{X}_{p}, \mathbf{Y}_{p^{\prime}}\right)=\frac{1}{i}\left\langle\int_{M} i_{\mathbf{X}_{p}}(\mathcal{B}) \mu_{\mathbf{X}_{p}}\left(p_{1}\right) \cdot \int_{M} \mathrm{P} \exp \left(\int_{p_{1}}^{p_{2}} A\right) i_{\mathbf{Y}_{p^{\prime}}}(\mathcal{C}) \mu_{\mathbf{Y}_{p^{\prime}}}\left(p_{2}\right)\right\rangle .
$$

Following a similar procedure as in the previous cases we get

$$
H_{A}\left(\mathbf{X}_{p}, \mathbf{Y}_{p^{\prime}}\right)=\sum_{j} \lambda_{j}^{-1} \int_{M} i_{\mathbf{X}_{p}}\left(\mathcal{B}_{j}\right) \mu_{\mathbf{X}_{p}}\left(p_{1}\right) \cdot \int_{M} \mathrm{P} \exp \left(\int_{p_{1}}^{p_{2}} A\right) i_{\mathbf{Y}_{p^{\prime}}}\left(\mathcal{C}_{j}\right) \mu_{\mathbf{Y}_{p^{\prime}}}\left(p_{2}\right)
$$

Using the same identities it yields

$$
H_{A}\left(\mathbf{X}_{p}, \mathbf{Y}_{p^{\prime}}\right)=\left.\left.\sum_{j} \lambda_{j}^{-1} \int_{M} \mathcal{B}_{j}\right|_{p_{1}} \wedge \eta_{\mathbf{X}_{p}}\left(p_{1}\right) \cdot \int_{M} \mathrm{P} \exp \left(\int_{p_{1}}^{p_{2}} A\right) \mathcal{C}_{j}\right|_{p_{2}} \wedge \eta_{\mathbf{Y}_{p^{\prime}}}\left(p_{2}\right)
$$

Using again the relation: $D_{A} \mathcal{C}_{j}=\lambda_{j} * \mathcal{B}_{j}$ and the completeness relation and integrating out rhs is rewritten as

$$
H_{A}\left(\mathbf{X}_{p}, \mathbf{Y}_{p^{\prime}}\right)=\int_{M} \operatorname{Tr} \mathrm{P} \exp \left(\oint_{\gamma} A\right) d \alpha_{\mathbf{X}_{p}} \wedge \alpha_{\mathbf{Y}_{p^{\prime}}}
$$

Take $\eta_{\mathbf{x}_{p}}$ and $\eta_{\mathbf{Y}_{p^{\prime}}}$, by Poincaré duality there are dual homology cycles $\Gamma_{\mathbf{X}_{p}}$ and $\Gamma_{\mathbf{Y}_{p^{\prime}}}$ respectively which are trivial homology classes, therefore $\gamma$ is a curve starting in $p_{2} \in W$ whose boundary is $\Gamma_{\mathbf{X}_{p}}$ to a point $u \in \Gamma_{\mathbf{Y}_{p^{\prime}}}$ completely contained in $\Gamma_{\mathbf{Y}_{p^{\prime}}}$. Then take a curve $\gamma_{0}$ from $u$ to $v$ in $\Gamma_{\mathbf{X}_{p}}$ and then go back to the point $p_{2}$ from $v$ contained completely in $W$.

Now consider de self-intersection i.e., take $\mathbf{X}_{p}=\mathbf{Y}_{p^{\prime}=p}$ to be a $p$-vector field and $H_{p}(M, \mathbb{R})=0$. The dimension of $M, n$ is $2 p+1$ then the expression reduces to

$$
H_{A}\left(\mathbf{X}_{p}, \mathbf{X}_{p}\right)=\int_{M} \operatorname{Tr} \mathrm{P} \exp \left(\oint_{\gamma} A\right) \alpha \wedge d \alpha
$$

We can think in this expression as the generalized asymptotic Hopf invariant associated to the field $\mathbf{X}_{p}$. 


\section{$5 \quad$ Higher Dimensional Asymptotic Jones-Witten In- variants}

We will give a briefly overview of the asymptotic Jones-Witten invariants [20], and give an extension to higher dimensions.

\subsection{Jones-Witten Invariants for Flows on 3-manifolds}

Let us consider a closed 3-manifold $M$ with a divergence-free vector field $X$ and an invariant probability measure $\mu$. Let $A$ be a connection on the $G$-principal bundle: $P \stackrel{\pi}{\rightarrow} M$. To define the asymptotic Jones-Witten invariant basically we will modify the the Wilson line and use the extended version, the asymptotic Wilson line. We will interpret this in terms of asymptotic homology cycles.

For the case of an abelian gauge group let us take $G=U(1)$, the asymptotic holon-

omy is the limit of the Wilson loop of $\operatorname{Hol}_{\widetilde{\Gamma}_{t, p}}(A)=P \exp \int_{\Gamma_{t, p}} A$, i.e. the asymptotic holonomy is defined as $\operatorname{Hol}_{\Gamma_{p}}(A)=\lim _{t \rightarrow \infty} \exp \int_{\tilde{\Gamma} t, p} \frac{1}{t} A$, next we will define the average holonomy of a $U(1)$-connection, over all the asymptotic cycles as

$$
\begin{aligned}
& \operatorname{Hol}_{X, \mu}(A)=\exp \int_{M} A(X) \mu \\
= & \lim _{t \rightarrow \infty} \exp \int_{M}\left(\int_{\widetilde{\Gamma}_{t, p}}\left(\frac{1}{t} A\right)\right) \mu(p) .
\end{aligned}
$$

For the non-abelian case, for instance, for $G=S U(2)$ Verjovsky and Vila Freyer redefine the asymptotic Wilson line in terms of the monodromy (see [20, 22] for details). In the present paper we will limited ourselves to the abelian case, thus we going come back to this case. In this case the asymptotic Jones-Witten invariants are defined by the following functional

$$
W_{X, \mu}(k)=\int_{\mathcal{A} / \mathcal{G}} \mathcal{D} A \exp \left(\frac{i k}{4 \pi} \int_{M} A \wedge d A\right) \cdot \operatorname{Hol}_{X, \mu}(A),
$$

where $k \in \mathbb{Z}, \mathcal{A}$ is the space of all $U(1)$-flat connections on $P$ and $\mathcal{G}$ the gauge group. The computation of $W_{X, \mu}(k)$ leads to [20, 22]:

$$
W_{X, \mu}(k)=c(M) \exp \left\{\frac{2 \pi i}{k} \int_{M} \alpha \wedge d \alpha\right\}
$$

where $c(M)$ contains the Ray-Singer torsion and the term of the exponential is precisely the asymptotic Hopf invariant, remember $i_{X}(\mu)=d \alpha$.

\subsection{Jones-Witten Invariants for a High Dimensional Flow}

In analogy to the previous section we define a Jones-Witten invariant for a flow in a $n=2 p+1$ dimensional manifold, with $H_{p}(M, \mathbb{R})=0$. Take a divergence-free $p$ vector field $\mathbf{X}_{p}$ and consider the $B F$ theory taking a $p$-form $B=C$ then the action is 
the Chern-Simons functional $S_{B F}=\int_{M} B \wedge d B$. We define the following asymptotic observable

$$
\mathcal{O}_{\mathbf{X}_{p}, \mu}=\exp \left(i \int_{M} i_{\mathbf{X}_{p}}(B) \mu\right) .
$$

Then the asymptotic Jones-Witten invariants in $n=2 p+1$ dimensions are given by

$$
\begin{gathered}
\mathcal{W}_{\mathbf{X}_{p}}(\mu):=\left\langle\mathcal{O}_{\mathbf{X}_{p}, \mu}\right\rangle \\
=\mathcal{N} \int \mathcal{D} B \exp \left(i \int_{M} B \wedge d B\right) \cdot \mathcal{O}_{\mathbf{X}_{p}, \mu}
\end{gathered}
$$

where $\mathcal{N}$ is the normalization factor.

As in previous cases we use the Hodge decomposition theorem and again only the transverse modes will contributes to the path integral. Recalling that $\mathbf{X}_{p}$ is a divergence-free vector field we have $i_{\mathbf{X}_{p}}(\mu)=d \alpha$. Then we have

$$
\mathcal{W}_{\mathbf{X}_{p}}(\mu)=\mathcal{N} \int \mathcal{D} B^{T} \exp \left(i \int_{M} B^{T} \wedge d B^{T}+B^{T} \wedge d \alpha^{\prime},\right)
$$

where $\alpha^{\prime}=(-1)^{\frac{p}{2}(3+p)} \alpha$.

The reason is the same as the previous case where the longitudinal and harmonic modes factor out and are absorbed in the normalization factor. Because the current (48) is closed in the de Rham sense (invariant under gauge transformation as in section 4) we take a basis to expand the transverse forms which satisfies $* d B_{j}=\lambda_{j} B_{j}$, where $\lambda_{j}^{2}$ are the eigenvalue of the laplacian. Expanding $B^{T}=\sum_{j} b_{j} B_{j}$, with $b_{j}$ 's being scalars and $\left\langle B_{i} \mid B_{k}\right\rangle=\int_{M} B_{i} \wedge * B_{k}=\delta_{i k}$. After some computations we get

$$
\mathcal{W}_{\mathbf{X}_{p}}(\mu)=\mathcal{N} \int \prod_{j} d b_{j} \exp \left(i \sum_{j} \lambda_{j}\left(b_{j}^{2}+\alpha_{j}^{\prime} b_{j}\right)\right),
$$

where $\alpha_{j}=\left\langle\alpha \mid B_{j}\right\rangle$. Finally we obtain

$$
\mathcal{W}_{\mathbf{X}_{p}}(\mu)=C(M) \exp \left(-\frac{i}{4} \sum_{j} \lambda_{j} \alpha_{j}^{\prime 2}\right),
$$

where $C(M)$ is a constant that can be removed by normalizing the expectation value. We observe that $\lambda_{j}\left\langle\alpha^{\prime} \mid B_{j}\right\rangle=\int_{M} d \alpha^{\prime} \wedge B_{j}$, where we did the integration, then the argument of the exponential is $\sum_{j} \int_{M} d \alpha^{\prime} \wedge B_{j} \int_{M} \alpha^{\prime} \wedge * B_{j}=\int_{M} \alpha \wedge d \alpha$, where we use the completeness relation and finally we get the expression

$$
\begin{gathered}
\mathcal{W}_{\mathbf{X}_{p}}(\mu)=C(M) \exp \left(-\frac{i}{4} \int_{M} \alpha \wedge d \alpha\right) \\
=C(M) \exp \left(-\frac{i}{4} H\left(\mathbf{X}_{p}\right)\right),
\end{gathered}
$$

where $H\left(\mathbf{X}_{p}\right)=H\left(\mathbf{X}_{p}, \mathbf{X}_{p}\right)$ is the high dimensional asymptotic Hopf invariant (33). The expression (53) is a topological invariant, i.e., the Jones-Witten invariants in $n=$ $2 p+1$ dimensions. They only depend on the flow $\mathbf{X}_{p}$ (or the foliation of the manifold $\mathrm{M}$, associated to the flow generated by $\mathbf{X}_{p}$ ) and the invariant measure $\mu$. 


\section{Asymptotic Invariants in String Theory}

It is known that $B F$ theories arises in natural way in supergravity in eleven dimensions. In addition to the Einstein-Hilbert action in eleven dimensions we have:

$$
S_{\text {sugra }}=\cdots+\int_{M_{11}}\left(G_{4} \wedge * G_{4}+C_{3} \wedge G_{4} \wedge G_{4}\right)+\text { anomalous terms }
$$

where $G_{4}=d C_{3}$. This is a theory with M2 branes $W$ coupled to the three-form $C_{3}$. The observables are the form $\int_{W} C_{3}$ and one can compute, for instance, the two-point correlation functions $\left\langle\int_{W} C_{3}(x) \cdot \int_{W} C_{3}(y)\right\rangle$

In Type II superstring theory we have the Chern-Simons coupling which is the coupling between the RR-fields $C_{p}$ and the other fields of the theory. In addition to the Dirac-Born-Infeld action we will have the CS action given by:

$$
\begin{gathered}
I_{C S}=\sum_{p} C_{p} \wedge \exp \left(2 \pi \alpha^{\prime}(B+F)\right) \\
=\int_{W_{p}} C_{p}+\int_{W_{p}} B \wedge C_{p-2}+\int_{W_{p}} F \wedge C_{p-2}+\cdots
\end{gathered}
$$

The action would be any of the two kinds of Type II theories [35] $S_{I I}$ contains a term of the CS form

$$
S_{I I A}=\cdots+\int_{M_{10}} B \wedge F_{4} \wedge F_{4}+\cdots
$$

where $F_{4}=d C_{3}$ is the field strength of $C_{3}$ or

$$
S_{I I B}=\cdots+\int_{M_{10}} C_{4} \wedge H_{3} \wedge F_{3}+\cdots
$$

where $H_{3}=d B$. In Type IIA $p=1,3,5,7,9$ and in Type IIB $p=0,2,4,6,8$. Thus we can define the correlation function corresponding to two non-intersecting D-branes of world-volumes $W$ and $W^{\prime}$ with RR-fields $C_{p}$ and $C_{p^{\prime}}$ respectively, thus we have

$$
\left\langle\int_{W} C_{p}(x) \cdot \int_{W^{\prime}} C_{p^{\prime}}(y)\right\rangle=\frac{\int \mathcal{D} C \exp \left[i S_{I I A, B}\right] \int_{W} C_{p}(x) \cdot \int_{W^{\prime}} C_{p^{\prime}}(y)}{\int \mathcal{D} C \exp \left[i S_{I I A, B}\right]}
$$

with $x \in W$ and $y \in W^{\prime}$. Of course the suitability of them depend on the possibility to solve the integration on the rhs. However in the present section we will not follow this path and we only study the consistency of the lhs and the definition of appropriated asymptotic observables. The observables $\int_{W} C_{p}$ are gauge invariant under gauge transformations $C_{p} \rightarrow C_{p}+d \Lambda_{p-1}$, they depend only of the homology class $[W]$ of $M$.

Now let us consider dynamical D-branes. It is known that one can scatter open or closed strings by D-branes but the D-branes as a dynamical objects also can be scattered by themselves. In the strong coupling limit $g_{S} \rightarrow \infty$, the D-branes are light objects and can be scattered by a center of forces [36]. In this section we would like

to study this system from the point of view of dynamical systems. To be more precise we describe the motion of a D-brane on a foliation $M_{1,9}=\mathbb{R} \times \mathbf{D}^{p} \times \mathbf{D}^{9-p}$, instead of 
a manifold $M_{1,9}=\mathbb{R} \times \mathbb{R}^{9}$. This corresponds physically to have a $\mathrm{D} p$-brane moving in the $M_{1,9}$ manifold along the transverse $(9-p)$ dimensions. In this case we can also define an asymptotic observable [16, 17, 18, 20] $\mathcal{O}_{\mathbf{X}}$ as:

$$
\mathcal{O}_{\mathbf{X}_{p}}=\int_{M} i_{\mathbf{X}_{p}}\left(C_{p}\right) \mu_{\mathbf{X}_{p}}
$$

Then the two-point correlation function is consequently

$$
\begin{gathered}
H\left(\mathbf{X}_{p}, \mathbf{Y}_{p^{\prime}}\right):=\frac{1}{i}\left\langle\mathcal{O}_{\mathbf{X}_{p}}(x) \cdot \mathcal{O}_{\mathbf{Y}_{p^{\prime}}}(y)\right\rangle \\
=\frac{1}{i}\left\langle\int_{M} i_{\mathbf{X}_{p}}\left(C_{p}\right) \mu_{\mathbf{X}_{p}}(x) \cdot \int_{M} i_{\mathbf{Y}_{p^{\prime}}}\left(C_{p^{\prime}}\right) \mu_{\mathbf{Y}_{p^{\prime}}}(y)\right\rangle .
\end{gathered}
$$

These quantities are invariant under the gauge transformations if the measures $\mu_{\mathbf{X}_{p}}$ and $\mu_{\mathbf{Y}_{p^{\prime}}}$ are invariant under the $p$ and $p^{\prime}$-flows respectively:

$$
\int_{M} i_{\mathbf{X}_{p}}\left(C_{p}\right) \mu_{\mathbf{X}_{p}}(x)=\int_{M} i_{\mathbf{X}_{p}}\left(C_{p}\right) \mu_{\mathbf{X}_{p}}(x)+\int_{M} i_{\mathbf{X}_{p}}\left(d \Lambda_{p-1}\right) \mu_{\mathbf{X}_{p}}(y),
$$

and similarly for $\mathbf{Y}_{p^{\prime}}$.

Again the second term vanishes by theorem 2A in [17]. One would try to interpret Eq. (60) as kind of linking number between the two flows $\mathbf{X}$ and $\mathbf{Y}$ associated to the propagation of the non-intersecting $\mathrm{D} p$ and $\mathrm{D} p^{\prime}$ branes in the spacetime.

Now let us consider a couple of non-intersecting D-branes, a $\mathrm{D} p$ and a $\mathrm{D} p^{\prime}$ of corresponding worldvolumes $W$ and $W^{\prime}$. The open string between the 2 D-branes is coupled to a closed string external background NS $B$-field and to a background abelian gauge connection $A$ with curvature $F=d A$. Within the Type II theory one can have the following observable

$$
\left\langle\int_{W} C_{p}(x) \cdot \int_{W^{\prime}} \exp \left(\int_{\Sigma} B-i \int d \sigma A_{I}(X) \partial_{\tau} X^{I}\right) C_{p^{\prime}}(y)\right\rangle,
$$

where $X$ is the embedding of the world-sheet $\Sigma$ into the target space manifold $M$, $\tau$ and $\sigma$ are the open string world-sheet coordinates and $I=0, \cdots, 9$. Of course in addition to the observables $\int_{W} C_{p}$ we have that the term $\int d \sigma A_{I}(X) \partial_{\tau} X^{I}$ is invariant under gauge transformations of the background connection $A \rightarrow A+d \lambda$. There is also a combined invariance under changes of $B$ and $A$ as follows: $\delta B_{I J}=\partial_{I} \Lambda_{J}-\partial_{J} \Lambda_{I}$ and $\delta A_{I}=-\Lambda_{I}$. (Compare with the Wilson surface introduced by Cattaneo and Rossi in Ref. 37.)

The corresponding asymptotic version is given by gauge invariant quantity

$$
\left\langle\int_{M} i_{\mathbf{X}_{p}}\left(C_{p}\right) \mu_{\mathbf{X}_{p}}(x) \cdot \int_{W^{\prime}} \exp \left(\int_{\Sigma} B-i \int d \sigma A_{I}(X) \partial_{\tau} X^{I}\right) i_{\mathbf{Y}_{p^{\prime}}}\left(C_{p^{\prime}}\right) \mu_{\mathbf{Y}_{p^{\prime}}}(y)\right\rangle .
$$

Just as in the case of $B F$ theory it is required that the above correlation function be gauge invariant. We can see that that is precisely the case if one of $\mathrm{D} p$ 's is and anti-Dbrane. The reason is as follows: it is well known that from the CS-action (55) that the 
RR-fields carries also $U(1)$-charge. Thus the field $C_{p}$ is charged under $U(1)$ as 1 while the $C_{p^{\prime}}$ transform as -1 . this implies that the whole observable and its correlation function will be gauge invariant. In a similar spirit to the $B F$ theories this quantity would compute some linking number of couple of non-intersecting flows two D-branes and anti-D-branes with a non-trivial phase given by the non-trivial class $\oint \frac{B}{2 \pi}$.

It is known that the true framework to deal D-branes is not cohomology but $\mathrm{K}$ theory. The description of D-branes in the set up of dynamical systems lead to the possibility of extending the asymptotic cycles as homology classes to K-homology cycles in K-theory.

\section{Final Remarks}

In the present paper we pursue the idea of the implementation of the procedure followed in Ref. [20] for Jones-Witten invariants, to compute link invariants for flows in higher dimensions. The relevant invariants of interest were elucidated in Refs. [15, 14]. We were able to obtain the higher-dimensional generalization of the asymptotic linking numbers for one flow (28) and for two flows (33). We also obtain the generalized linking numbers in the non-abelian case for one flow (39) and two flows (44). Therefore for all these mentioned cases we were able to associate a link invariant in higher dimensions to a flow (or flows). We calculate the linking number for a flow (foliation), with this we were able to find the higher dimensional $n=2 p+1$ generalization of asymptotic Hopf invariant and consequently of the Jones-Witten invariants for flows (53) considered in [20] for the three dimensional case. Finally some speculations about a way to incorporate asymptotic cycles was discussed in Sec. (6) and we found that one condition to find gauge invariant correlation functions of observables constructed with RR-fields, $B$-fields and gauge fields impose that the flows associated to the D-branes correspond to a pair $\mathrm{D} p$ - $\overline{\mathrm{D}} p^{\prime}$-brane (pair brane-antibrane). Rather than cohomology, RR-fields take values in $K$-theory, thus it would be interesting to carry over the construction of asymptotic cycles to $K$-homology. Some of this work is in progress.

To find these invariants we modify only the observables which have the information of the flow. These observables were constructed with the ideas of asymptotic cycles, geometric currents and foliations introduced by Sullivan [19] and Schwarzman [17]. Thus we take the asymptotic observables and the path integral give the asymptotic linking number a (Hopf invariant or helicity). Other invariants of knots and links in the context of Batalin-Vilkovisky (BV) quantization incorporating Wilson surfaces [37] and string topology [38] are our strong interest and will be considered in a future publication. Moreover a generalized asymptotic linking number (Sec. 4) would be extended by considering a $p$-form gauge potential [39].

On the other hand, in other theories as the AdS/CFT correspondence, there is also an underlying $B F$ theory of the form $\int_{Y} B_{R R} \wedge d B_{N S}$ [40]. Thus the observables of the theory are also susceptible to be extended as there exist a flow determined by a vector field $\mathbf{X}$. Moreover, the Hitchin functional $\int_{M_{7}} \Phi \wedge \star_{7} \Phi$ is defined on a 7 manifold $M_{7}$ of $G_{2}$-holonomy, which resembles a $B F$ action. It is worth to mention that the partition function at one-loop has been computed recently [41, 42] in terms of the BV 
formalism by obtaining the Ray-Singer torsion of $M_{7}$. It would be interesting to find the observables of the theory and their asymptotic counterparts. Some of the results on this subject will be reported elsewhere.

\section{Acknoledgements}

It is a pleasure to thank B. Itzá, A. Martínez-Merino, P. Paniagua and A. Pedroza for enlightening discussions and useful suggestions. This work of R.S. is supported in part by CONACyT graduate fellowship.

\section{References}

[1] E. Witten, Commun. Math. Phys. 117, 353 (1988).

[2] E. Witten, Math. Res. Lett. 1769 (1994).

[3] E. Witten, Commun. Math. Phys. 118, 411 (1988).

[4] A.S. Schwarz, Lett. Math. Phys. 2, 247 (1978); Commun. Math. Phys. 67, 1 (1979).

[5] D.B. Ray and I.M. Singer, Adv. Math. 7, 145 (1971).

[6] E. Witten, Commun. Math. Phys. 121, 351 (1989).

[7] M. Atiyah, The Geometry and physics of knots, Cambridge, UK: Univ. Pr. (1990) $78 \mathrm{p.}$

[8] D. Birmingham, M. Blau, M. Rakowski and G. Thompson, Phys. Rept. 209, 129 (1991).

[9] A. S. Schwarz, Quantum field theory and topology, Berlin, Germany: Springer (1993) $274 \mathrm{p}$.

[10] L. Rozansky and E. Witten, arXiv:hep-th/9612216].

[11] J.M. Labastida and M. Mariño, Topological Quantum Field Theory and Four Manifolds, Springer Verlag (2005).

[12] M. Mariño, Chern-Simons theory, matrix models, and topological strings, Oxford, UK: Clarendon (2005) 197 p; "Chern-Simons theory and topological strings," Rev. Mod. Phys. 77, 675 (2005) arXiv:hep-th/0406005.

[13] G. T. Horowitz, Commun. Math. Phys. 125, 417 (1989).

[14] M. Blau and G. Thompson, Annals Phys. 205, 130 (1991).

[15] G. T. Horowitz and M. Srednicki, "A Quantum Field Theoretic Description of Linking Numbers and their Generalization," Commun. Math. Phys. 130, 83 (1990). 
[16] S. Schwartzman, "Asymptotic cycles", Ann. Math. 66, 270-284 (1957).

[17] S. Schwartzman, "Higher Dimensional Asymptotic Cycles", Canad. J. Math. 55(3), 636-648 (2003).

[18] D. Ruelle and D. Sullivan, "Currents, Flows and Diffeomorphisms", Topology 14 319-327 (1975).

[19] D. Sullivan, "Cycles for the Dynamical Study of Foliated Manifolds and Complex Manifolds", Invent. Math. 36 225-255 (1976).

[20] A. Verjovsky, R. Vila Freyer, "The Jones-Witten Invariant for Flows on a 3Dimensional Manifold", Commun. Math. Phys. 163 73-88 (1994).

[21] V.I. Arnold, "The Asymptotic Hopf Invariant and its Applications", Materialy Vsesoyuznoi Shkoly po Differentsialnym Uravneniyam s Beskonechnym Chislom Nezavisimyh i po Dynamicheskim Sistemam c Beskonechnym (in russian), 197374, English transl. in Sel. Math. Sov. 5, 327-345 (1986).

[22] V.I. Arnold and B.A. Khesin, Topological Methods in Hydrodynamics, Springer Verlag (1998).

[23] T. Vogel, "On the Asymptotic Linking Number", Proc. Am. Math. Soc. 131, 2289-2297 (2002).

[24] L. Woltjer, Proc. Nat. Acad. Sci. USA 44 (1958) 489-491; H.K. Moffat, J. Fluid Mech. 159 (1985) 359-378; J. Fluid Mech. 166 (1986) 359-378.

[25] D. Kotschick and T. Vogel, "Linking Numbers of Measured Foliations", Ergodic Theory Dynam. Systems, 23 541-558 (2003).

[26] S. Baader, "Asymptotic link invariants for ergodic vector fields", arXiv:0803.0898 [math.GT].

[27] S. Baader, "Asymptotic Vassiliev Invariants for Vector Fields", arXiv:0810.3870 [math.GT].

[28] E. Ghys, "Knots and Dynamics", "Proceedings of the International Congress of Mathematicians, vol. I, Madrid, 2006.

[29] Y. S. Wu and A. Zee, Phys. Lett. B 207 (1988) 39.

[30] A. M. Polyakov, Mod. Phys. Lett. A 3, 325 (1988).

[31] G. de Rham, Differentiable Manifolds: Forms, Currents, Harmonic Forms, Springer-Verlag Berlin Heidelberg 1984.

[32] M. Holm, "New insights in brane and Kaluza-Klein theory through almost product structures", arXiv:hep-th/9812168v1. 
[33] I. Vaisman, Lectures on the Geometry of Poisson Manifolds, (Boston, MA: Birkhauser, 1994).

[34] R. Bott and L.W. Tu, Differential Forms in Algebraic Topology, Springer Verlag, New York, 1986.

[35] J. Polchinski, "String theory. Vol. 2: Superstring theory and beyond," Cambridge, UK: Univ. Pr. (1998) $531 p$

[36] L. McAllister and I. Mitra, JHEP 0502, 019 (2005) arXiv:hep-th/0408085.

[37] A. S. Cattaneo and C. A. Rossi, Commun. Math. Phys. 256, 513 (2005) arXiv:math-ph/0210037.

[38] A. S. Cattaneo, J. Frohlich and B. Pedrini, Commun. Math. Phys. 240, 397 (2003) arXiv:math/0202176.

[39] M. Henneaux and C. Teitelboim, Found. Phys. 16, 593 (1986).

[40] E. Witten, JHEP 9812, 012 (1998) arXiv:hep-th/9812012].

[41] V. Pestun and E. Witten, Lett. Math. Phys. 74, 21 (2005) arXiv:hep-th/0503083].

[42] J. de Boer, P. de Medeiros, S. El-Showk and A. Sinkovics, Class. Quant. Grav. 25, 075006 (2008) [arXiv:0706.3119 [hep-th]]. 